PROCEEDINGS OF THE

AMERICAN MATHEMATICAL SOCIETY

Volume 138, Number 6, June 2010, Pages 2095-2098

S 0002-9939(10)10233-0

Article electronically published on January 29, 2010

\title{
A NOTE ON THE BILINEAR LITTLEWOOD-PALEY SQUARE FUNCTION
}

\author{
PARASAR MOHANTY AND SAURABH SHRIVASTAVA
}

(Communicated by Michael T. Lacey)

\begin{abstract}
In this paper, we give an elementary proof of boundedness of the smooth bilinear Littlewood-Paley square function.
\end{abstract}

\section{INTRODUCTION}

The study of bilinear multiplier operators has recently received a great deal of attention with the work of Lacey and Thiele [4, [5. They proved the boundedness of the bilinear Hilbert transform and established the answer to a long-standing conjecture of A.P. Calderón.

One of the important themes in the study of linear multipliers is Rubio de Francia's result on Littlewood-Paley square functions [7]. His result is:

$$
\left\|\left(\sum_{j}\left|S_{I_{j}} f\right|^{2}\right)^{\frac{1}{2}}\right\|_{p} \leq C\|f\|_{p}
$$

for $p \geq 2$, where $S_{I_{j}} f=\left(\chi_{I_{j}} \hat{f}\right)^{\vee}$ and the $I_{j}$ 's are disjoint intervals. First, Lacey 2 addressed this problem in the bilinear setting. Later, Bernicot [1] proved a version of Rubio de Francia's result in the bilinear case. The proofs of these two results are very intricate. In 2 2 Lacey posed a problem regarding the validity of the boundedness of smooth square functions for certain values of $p$. Surprisingly, the answer to his question can be given even without any use of time-frequency analysis. The purpose of this note is to give an answer to Lacey's question.

Let $K$ be a smooth bump function defined on $\mathbb{R}^{d}$ such that $\hat{K}$ is supported in the unit cube of $\mathbb{R}^{d}$. For $n \in \mathbb{Z}^{d}$, let $K_{n}$ be the function defined by $\hat{K}_{n}(\xi)=\hat{K}(\xi-n)$. For $f, g \in \mathcal{S}\left(\mathbb{R}^{d}\right)$ consider the bilinear operator

$$
S_{n}(f, g)(x)=\int_{\mathbb{R}^{d}} f(x-y) g(x+y) K_{n}(y) d y .
$$

Let $S(f, g)$ denote the bilinear Littlewood-Paley square function associated with this sequence of operators, i.e.

$$
S(f, g)(x)=\left(\sum_{n}\left|S_{n}(f, g)(x)\right|^{2}\right)^{\frac{1}{2}} .
$$

In 2] Lacey proved the following result:

Received by the editors September 25, 2009.

2000 Mathematics Subject Classification. Primary 42A45, 42B15, $42 \mathrm{~B} 25$.

Key words and phrases. Bilinear multiplier operators, Littlewood-Paley square function.

(C)2010 American Mathematical Society

Reverts to public domain 28 years from publication 
Theorem $1.1([2])$. For all $2 \leq p_{1}, p_{2} \leq \infty$ with $\frac{1}{p_{1}}+\frac{1}{p_{2}}=\frac{1}{2}$,

$$
\|S(f, g)\|_{2} \leq c\|f\|_{p_{1}}\|g\|_{p_{2}}
$$

where the constant $C$ depends on the function $K$.

The problem for nonsmooth symbols remained open for a long time. Recently Bernicot 1 addressed this problem and obtained the boundedness of the nonsmooth Littlewood-Paley square function associated with a sequence of intervals satisfying certain conditions. In particular, the bilinear analogue of Carleson's square function has been obtained.

In the proof of Theorem 1.1 the exponent 2, the decay of the bump function $K$, and the support condition on $\hat{K}$ play important roles. In [2] the author posed a question about the boundedness of $S(f, g)$ into $L^{p_{3}}\left(\mathbb{R}^{d}\right)$ for exponents $p_{3}>2$. The answer follows from an adaptation of the techniques used by Rubio de Francia in [6. Moreover, with this technique one gets the result for a much wider range of the exponent $p_{3}$ and with weaker restrictions on $K$. Also, we will show that $p_{1}, p_{2} \geq 2$ is a necessary condition. But still we do not have any idea about the sharpness of the bound for the exponent $p_{3}$.

\section{Smooth Bilinear Littlewood-Paley square FUnction}

In this section we obtain the result of Theorem 1.1 for a larger range of exponents $p_{1}, p_{2}$, and $p_{3}$ using the ideas from [6].

Theorem 2.1. Let $m \in \mathcal{S}(\mathbb{R})$. For $k \in \mathbb{Z}$ define $m_{k}(\xi)=m(\xi-k)$ and let $S_{k}$ be the bilinear multiplier operator associated with $m_{k}$. Then for $2<p_{1}, p_{2} \leq \infty$ and $4 / 3<p_{3} \leq \infty$ satisfying $\frac{1}{p_{1}}+\frac{1}{p_{2}}=\frac{1}{p_{3}}$, we have

$$
\|S(f, g)\|_{p_{3}} \leq C\|f\|_{p_{1}}\|g\|_{p_{2}} .
$$

Proof. In order to prove inequality (2.1), it is enough to prove that for almost every $x \in \mathbb{R}, S(f, g)(x)$ satisfies the pointwise estimate

$$
S(f, g)(x) \leq C\left(M\left(|f|^{2},|g|^{2}\right)(x)\right)^{\frac{1}{2}}=C M_{2}(f, g)(x)
$$

where $M$ is the bilinear Hardy-Littlewood maximal operator, given by

$$
M(f, g)(x)=\sup _{x \in I} \frac{1}{|I|} \int_{I}|f(x-y) g(x+y)| d y,
$$

and $C$ is a constant independent of $f$ and $g$. The above estimate gives us the desired result upon using the boundedness of the bilinear Hardy-Littlewood maximal operator $M$ proved by Lacey in [3], which states that $\|M(f, g)\|_{p_{3}} \leq C\|f\|_{p_{1}}\|g\|_{p_{2}}$ where $\frac{1}{p_{3}}=\frac{1}{p_{1}}+\frac{1}{p_{2}}$ and $2 / 3<p_{3} \leq \infty, 1<p_{1}, p_{2} \leq \infty$.

Let $a=\left\{a_{k}\right\}$ be a finite sequence in $l_{2}(\mathbb{Z})$ with norm one. Then we prove that for a.e. $x \in \mathbb{R}$,

$$
\left|\sum_{k} a_{k} S_{k}(f, g)(x)\right| \leq C M_{2}(f, g)(x),
$$


which gives the claimed estimate (2.2) for the square function $S(f, g)$. Consider

$$
\begin{aligned}
\sum_{k} a_{k} S_{k}(f, g)(x) & =\sum_{k} a_{k} \int_{\mathbb{R}} f(x-y) g(x+y) \check{m_{k}}(y) d y \\
& =\int_{\mathbb{R}} f(x-y) g(x+y) \check{m}(y) \sum_{k} a_{k} e^{2 \pi i k y} d y \\
& =\int_{\mathbb{R}} f(x-y) g(x+y) \check{m}(y) h(y) d y,
\end{aligned}
$$

where $h$ is the Fourier transform of the sequence $a$ and is a periodic function with unit $L^{2}(I)$ norm, for $I=[-1 / 2,1 / 2]$. Hence

$$
\begin{aligned}
\sum_{k} a_{k} S_{k}(f, g)(x) & =\int_{\mathbb{R}} f(x-y) g(x+y) \check{m}(y) h(y) d y \\
& =\left(\int_{I}+\sum_{n=1}^{\infty} \int_{2^{n} I \backslash 2^{n-1} I}\right) f(x-y) g(x+y) \check{m}(y) h(y) d y .
\end{aligned}
$$

Since $m \in \mathcal{S}(\mathbb{R})$, there exists a constant $C_{N}$ such that $|\check{m}(y)| \leq \frac{C_{N}}{(1+|y|)^{N}}$ for all $N \in \mathbb{N}$. Using this decay estimate for $\check{m}$ and Hölder's inequality, we obtain

$$
\begin{aligned}
\left|\sum_{k} a_{k} S_{k}(f, g)(x)\right| & \leq C_{N} \sum_{n=0}^{\infty} 2^{-n N} 2^{n / 2}\left(\int_{2^{n} I}|f(x-y) g(x+y)|^{2} d y\right)^{1 / 2} \\
& \leq C_{N} \sum_{n=0}^{\infty} 2^{-n(N-1)}\left(M\left(|f|^{2},|g|^{2}\right)(x)\right)^{\frac{1}{2}} \\
& \leq C_{N} M_{2}(f, g)(x) .
\end{aligned}
$$

This completes the proof in one dimension.

Note that this result covers a wider range of exponents than mentioned by Lacey in dimension 1. If the dimension $d>1$, then using the same idea we obtain that the above theorem holds when $p_{3} \geq 2$ and $2<p_{1}, p_{2} \leq \infty$, as the $d$-dimensional bilinear Hardy-Littlewood maximal operator $M_{2}$ is easily seen to be bounded for the above-mentioned range as a consequence of Hölder's inequality and interpolation. So, this completely answers the question posed by Lacey.

We would like to make some remarks:

(1) In the proof of Theorem 2.1 we only need a $\frac{C}{(1+|y|)^{2}}$ type decay for the function $\check{m}$, and no support condition on $m$ is required.

(2) For the case where $p_{3}=2$ and either of $p_{1}, p_{2}$ is 2 , this technique will yield that the operator $S$ is of weak type as the operator $M_{2}$ is of weak type for these exponents. However, Lacey's result, Theorem 1.1, provides better estimates for these end-point cases when $m$ has compact support.

Now we shall show that $p_{1}, p_{2} \geq 2$ is a necessary condition in Theorem 2.1. This can be achieved using a well known example adapted to our setting.

Proposition 2.2. If Theorem 2.1 holds true for some indices $p_{1}, p_{2}, p_{3}$, then it is a necessary condition that $p_{1}, p_{2} \geq 2$. 
Proof. Let $\hat{f}_{N}=\chi_{[0, N]}, \hat{g}=\chi_{[0,1 / 2]}$ and $m \in \mathcal{S}(\mathbb{R})$ be such that $\operatorname{supp}(m) \subseteq[0,1]$. Observe that

$$
S_{k}\left(f_{N}, g\right)(x)=\int_{\mathbb{R}} \int_{\mathbb{R}} \chi_{[0, N]}(\xi-\eta) \chi_{[0,1 / 2]}(\eta) m(\xi-2 \eta-k) d \eta e^{2 \pi i \xi x} d \xi .
$$

An easy computation will show that for $1 \leq k \leq N-2$ and $\eta \in[0,1 / 2], \xi-2 \eta-k \in$ $[0,1]$ implies that $\xi-k \in[0,2]$ and $\xi-\eta \in[0, N]$. Hence, for $1 \leq k \leq N-2$, we have

$$
\begin{aligned}
\widehat{S_{k} \widehat{\left(f_{N}, g\right)}(\xi)} & =\int_{\mathbb{R}} \chi_{[0, N]}(\xi-\eta) \chi_{[0,1 / 2]}(\eta) m(\xi-2 \eta-k) d \eta \\
& =\int_{0}^{1 / 2} \chi_{[0, N]}(\xi-\eta) m(\xi-2 \eta-k) d \eta \\
& =\frac{1}{2} \chi_{[0,2]}(\xi-k) \int_{0}^{1} m(\xi-\eta-k) d \eta \\
& =\hat{F}(\xi-k),
\end{aligned}
$$

where $\hat{F}(\xi)=\frac{1}{2} \chi_{[0,2]}(\xi) \int_{0}^{1} m(\xi-\eta) d \eta$. Thus for $1 \leq k \leq N-2$, we have

$$
\left|S_{k}\left(f_{N}, g\right)(x)\right|=|F(x)| \text {. }
$$

If Theorem 2.1 holds true, then we have that

$$
N^{1 / 2} \approx\left\|\left(\sum_{k=1}^{N-2}\left|S_{k}\left(f_{N}, g\right)(x)\right|^{2}\right)^{1 / 2}\right\|_{p_{3}} \leq\left\|S\left(f_{N}, g\right)\right\|_{p_{3}} \leq C\left\|f_{N}\right\|_{p_{1}}\|g\|_{p_{2}} .
$$

This implies that

$$
N^{1 / 2} \leq C^{\prime} N^{1 / p_{1}^{\prime}}
$$

where the constant $C^{\prime}$ does not depend on $N$. Hence we get that $p_{1} \geq 2$. Similar arguments can be given to show that $p_{2}$ also satisfies the same condition.

\section{REFERENCES}

[1] Bernicot, F., $L^{p}$ estimates for non smooth bilinear Littlewood-Paley square functions on $\mathbb{R}$, arXiv:0811.2854, to appear in Math. Ann.

[2] Lacey, M., On bilinear Littlewood-Paley square functions, Publ. Mat. 40 (1996), no. 2, 387396. MR.1425626 (98c:42017)

[3] Lacey, M., The bilinear maximal functions map into $L^{p}$ for $2 / 3<p \leq 1$, Ann. of Math. (2) 151 (2000), no. 1, 35-57. MR1745019 (2001b:42015)

[4] Lacey, M., Thiele, C., $L^{p}$ estimates on the bilinear Hilbert transform for $2<p<\infty$, Ann. of Math. (2) 146 (1997), no. 3, 693-724. MR.1491450 (99b:42014)

[5] Lacey, M., Thiele, C., On Calderón's conjecture, Ann. of Math. (2) 149 (1999), no. 2, 475-496. MR1689336 (2000d:42003)

[6] Rubio de Francia, J. L., Estimates for some square functions of Littlewood-Paley type, Publ. Sec. Mat. Univ. Autònoma Barcelona 27 (1983), no. 2, 81-108. MR.765844 (86d:42018)

[7] Rubio de Francia, J. L., A Littlewood-Paley inequality for arbitrary intervals, Rev. Mat. Iberoamericana 1 (1985), no. 2, 1-14. MR850681 (87j:42057)

Department of Mathematics and Statistics, Indian Institute of Technology Kanpur, KANPUR-208016, INDIA

E-mail address: parasar@iitk.ac.in

Department of Mathematics and Statistics, Indian Institute of Technology Kanpur, KANPUR-208016, INDIA

E-mail address: saurabhk@iitk.ac.in 